\title{
Correction to: Ideology and non-state climate action: partnering and design of REDD+ projects
}

\section{Benjamin M. Abraham ${ }^{1}$}

Published online: 8 July 2021

(c) The Author(s) 2021

\section{Correction to: Int Environ Agreements https://doi.org/10.1007/s10784-021-09537-4}

In the original publication of the article, Tables 2 and 3 and their corresponding citations were incorrectly displayed. This has been corrected with this Correction.

The original article has been corrected.

The original article can be found online at https://doi.org/10.1007/s10784-021-09537-4.

Benjamin M. Abraham

abrahambm@gmail.com

1 Blavatnik School of Government, University of Oxford, Radcliffe Observatory Quarter, Woodstock Road, Oxford OX2 6GG, UK 
Table 2 Expected observable implications for relationships between environmental worldviews and aspects of project design

\begin{tabular}{llllll}
\hline & Social benefits & $\begin{array}{l}\text { Ecological } \\
\text { benefits }\end{array}$ & $\begin{array}{l}\text { Market } \\
\text { financing }\end{array}$ & $\begin{array}{l}\text { Non-market } \\
\text { financing }\end{array}$ & Local MRV \\
\hline Market liberal & - & - & + & $\mathrm{n} / \mathrm{a}$ & - \\
Bioenvironmentalist & $\mathrm{n} / \mathrm{a}$ & + & - & $\mathrm{n} / \mathrm{a}$ & $\mathrm{n} / \mathrm{a}$ \\
Social green & + & $\mathrm{n} / \mathrm{a}$ & - & + & + \\
\hline
\end{tabular}

Table 3 Summary of model variables

\begin{tabular}{|c|c|c|c|c|c|}
\hline Variables & Obs & Mean & SD & Min & $\operatorname{Max}$ \\
\hline \multicolumn{6}{|l|}{ Design aspects } \\
\hline Social benefits & 365 & 0.68 & 0.47 & 0 & 1 \\
\hline Ecological benefits & 366 & 0.54 & 0.50 & 0 & 1 \\
\hline Market financing & 356 & 0.82 & 0.38 & 0 & 1 \\
\hline Non-market financing & 339 & 0.50 & 0.50 & 0 & 1 \\
\hline Local MRV & 292 & 0.30 & 0.46 & 0 & 1 \\
\hline \multicolumn{6}{|l|}{ Environmental worldviews } \\
\hline$\%$ Market liberal & 387 & 50.5 & 36.4 & 0 & 100 \\
\hline$\%$ Bioenvironmentalist & 387 & 15.8 & 25.4 & 0 & 100 \\
\hline$\%$ Social green & 387 & 10.4 & 21.6 & 0 & 100 \\
\hline \multicolumn{6}{|l|}{ Actor types } \\
\hline$\%$ Host private sector & 385 & 14.6 & 27.2 & 0 & 100 \\
\hline$\%$ Int'l private sector & 387 & 21.9 & 30.5 & 0 & 100 \\
\hline$\% \mathrm{MDB}$ & 385 & 3.40 & 11.9 & 0 & 100 \\
\hline$\%$ Host subnational public & 385 & 7.31 & 17.0 & 0 & 100 \\
\hline$\%$ Host national public & 385 & 9.71 & 18.8 & 0 & 100 \\
\hline$\%$ Int'l national public & 385 & 5.17 & 11.8 & 0 & 50 \\
\hline$\%$ IGO & 385 & 1.67 & 6.57 & 0 & 50 \\
\hline$\%$ Host NGO & 385 & 15.3 & 23.2 & 0 & 100 \\
\hline$\%$ Int'1 NGO & 385 & 15.7 & 23.7 & 0 & 100 \\
\hline$\%$ University/research & 385 & 3.22 & 9.92 & 0 & 75 \\
\hline \multicolumn{6}{|l|}{ Controls } \\
\hline Private land tenure & 315 & 0.38 & 0.49 & 0 & 1 \\
\hline State land tenure & 315 & 0.57 & 0.50 & 0 & 1 \\
\hline Community land tenure & 315 & 0.19 & 0.40 & 0 & 1 \\
\hline Protected area & 382 & 0.31 & 0.46 & 0 & 1 \\
\hline EPI biohabitat score & 370 & 81.5 & 11.8 & 31.9 & 98.6 \\
\hline Civil liberties & 370 & 3.41 & 1.31 & 1.00 & 6.00 \\
\hline GDP per capita & 370 & 8.54 & 0.96 & 6.35 & 10.4 \\
\hline
\end{tabular}

Publisher's Note Springer Nature remains neutral with regard to jurisdictional claims in published maps and institutional affiliations. 